\title{
On Integral Sum Numbers of Cycles
}

\author{
Ergen Liu, Qing Zhou, and Wei Yu \\ School of Basic Sciences, East China Jiaotong University \\ Nanchang, jiangxi China, 330013 \\ liueg65@126.com, leg_eg@sina.com
}

\begin{abstract}
In this paper we determine that integral sum number of graph $C_{n}$, namely for any integer $n \geq 5$, then $\xi\left(C_{n}\right)=0$, therefore we prove that the graph $C_{n}(n \geq 5)$ is an integral sum graph.
\end{abstract}

Keywords: Integral sum number, Integral sum graph, Graph $C_{n}$.

\section{Introduction}

The graph in this paper discussed are undirected, no multiple edges and simple graph, the unorganized state of definitions and terminology and the symbols in this paper referred to reference [1],[2].

F. Harary [3] introduce the concept of integral sum graphs. The integral sum graph $G^{+}(S)$ of a finite subset $S \subset Z$ is the graph $(V, E)$, where $V=S$ and $u v \in E$ if and only if $u+v \in S$. A graph $G$ is an integral sum graph if it is isomorphic to the integral sum graph number of $G^{+}(S)$ of some $S \subset Z$. The integral sum number of a given graph $G$, denoted by $\xi(G)$, is defined as the smallest nonnegative integer S such that $G \bigcup s k_{1}$ is an integral sum graph. For convincing, an integral sum graph is written as an integral sum graph in references [3,4]. Obviously, graph $G$ is an integral sum graph if $\xi(G)=0$.

It is very difficult to determine $\xi(G)$ for a given graph $G$ in general. All paths and matchings are verified to be integral sum graph in references [3], and we see from references [4] that $\xi\left(C_{n}\right) \leq 1$ for all $n \neq 4$. And further, an open conjecture was posed in references [4] as follows:

Conjecture [4]: Is it true that any old cycle is an integral sum graph?

Definition 1.1. If a graph is isomorphism graph $G^{+}(S)$, then we call graph $G$ is Integral sum graph, denoted by $G \cong G^{+}(S)$. 
Definition 1.2. For graph $G$, if it exists nonnegative integer $S$ such that $G \bigcup s k_{1}$ is an integral sum graph, then we call number $s$ is integral sum number of $G$, denoted by $\xi(G)=s$.

\section{Main Results and Certification}

Theorem 2.1. For any integer $n \geq 3$, then

$$
\xi\left(C_{n}\right)=\left\{\begin{array}{l}
3, \text { when } n=4 \\
0, \text { when } n \neq 4
\end{array}\right.
$$

Proof. It is immediate from references [3, 4] that $\xi\left(C_{3}\right)=0$ and $\xi\left(C_{4}\right)=3$. And it is clear that $C_{5} \cong G^{+}\{2,1,-2,3,-1\}$ and $C_{7} \cong G^{+}\{1,2,-5,7,-3,4,3\}$.

Next we consider two cases: For all $C_{2 j}(j \geq 3)$ and $C_{2 j+1}(j \geq 4)$, we will show that the two classes of cycle are integral sum graph.

Let the vertices of $C_{2 j}(j \geq 3)$ and $C_{2 j+1}(j \geq 4)$ be marked as the methods in Fig.1 and Fig.2.

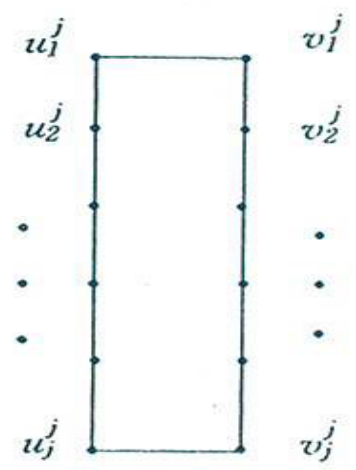

Fig. 1. Marking of $C_{2 j}$

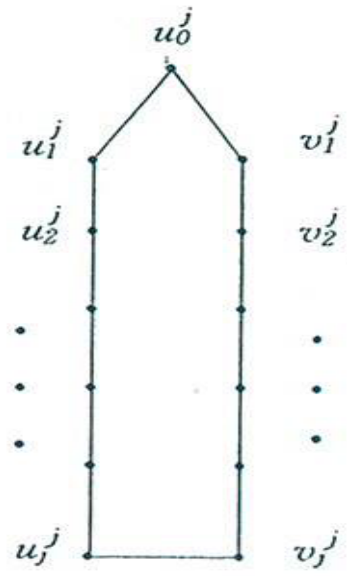

Fig. 2. Marking of $C_{2 j+1}$

\section{Case 1. When $n=2 j(j \geq 3)$}

We first give the labels of $C_{6}$ and $C_{8}$ as follows:

Let $u_{1}^{3}=4, u_{2}^{3}=-1, u_{3}^{3}=5 ; v_{1}^{3}=1, v_{2}^{3}=3, v_{3}^{3}=-2$.

and

$$
u_{1}^{4}=7, u_{2}^{4}=-2, u_{3}^{4}=9, u_{4}^{4}=-11 ; v_{1}^{4}=2, v_{2}^{4}=5, v_{3}^{4}=-3, v_{4}^{4}=8
$$


Then $C_{6} \cong G^{+}\{4,-1,5,-2,3,1\}$ and $C_{8} \cong G^{+}\{7,-2,9,-11,8,-3,5,2\}$, therefore $\xi\left(C_{6}\right)=0$ and $\xi\left(C_{8}\right)=0$.

When $j \geq 5$, we give the labels of $C_{2 j}$ as follows:

Let $\quad u_{1}^{j}=u_{1}^{j-1}+u_{1}^{j-2} \quad(j \geq 5) ; \quad u_{2}^{j}=u_{2}^{j-1}+u_{2}^{j-2} \quad(j \geq 5) ;$

$$
v_{1}^{j}=v_{1}^{j-1}+v_{1}^{j-2} \quad(j \geq 5) ; \quad v_{2}^{j}=v_{2}^{j-1}+v_{2}^{j-2} \quad(j \geq 5) .
$$

and

$$
\begin{aligned}
& u_{k}^{j}=u_{k-2}^{j}-u_{k-1}^{j} \quad(k=3,4, \cdots, j) ; \\
& v_{k}^{j}=v_{k-2}^{j}-v_{k-1}^{j} \quad(k=3,4, \cdots, j) .
\end{aligned}
$$

By labeling of above, we know $\xi\left(C_{2 j}\right)=0(j \geq 5)$.

The labeling of above is illustrated in Fig.3.
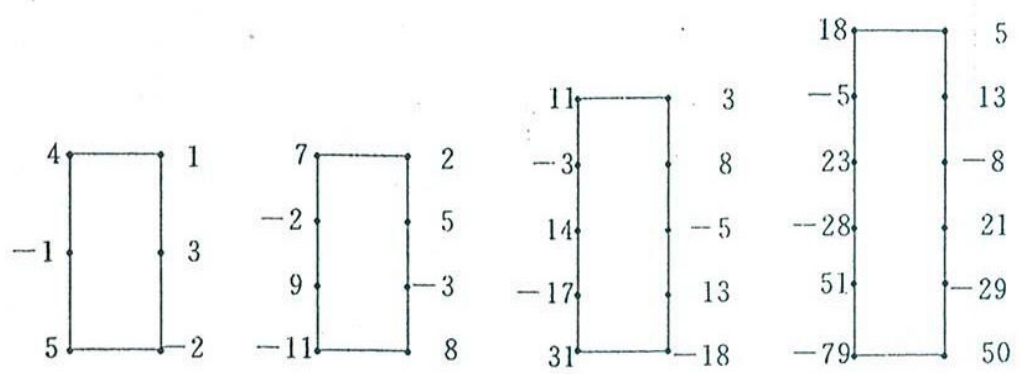

Fig. 3. Labeling of $C_{2 j}(j \geq 3)$

Case 2. When $n=2 j+1(j \geq 4)$

We first give the labels of $C_{9}$ and $C_{11}$ as follows:

Let $u_{0}^{4}=2, u_{1}^{4}=5, u_{k}^{4}=u_{k-2}^{4}-u_{k-1}^{4}(k=2,3,4)$;

$$
v_{1}^{4}=-5, v_{2}^{4}=7, v_{k}^{4}=v_{k-2}^{4}-v_{k-1}^{4}(k=3,4) .
$$

and

$$
\begin{aligned}
& u_{0}^{5}=3, u_{1}^{5}=8, u_{k}^{5}=u_{k-2}^{5}-u_{k-1}^{5}(k=2,3,4,5) ; \\
& v_{1}^{5}=-8, v_{2}^{5}=11, v_{k}^{5}=v_{k-2}^{5}-v_{k-1}^{5}(k=3,4,5) .
\end{aligned}
$$

Then $C_{9} \cong G^{+}\{2,5,-3,8,-11,19,-12,7,-5\}$ and $C_{11} \cong G^{+}\{3,8,-5,13,-18,31,-49,30,-19,11,-8\}$, therefore $\xi\left(C_{9}\right)=0$ and $\xi\left(C_{11}\right)=0$.

When $j \geq 6$, we give the labels of $C_{2 j+1}$ as follows:

$$
\text { Let } \begin{aligned}
u_{0}^{j} & =u_{0}^{j-1}+u_{0}^{j-2} \quad(j \geq 6) ; \quad u_{1}^{j}=u_{1}^{j-1}+u_{1}^{j-2} \quad(j \geq 6) ; \\
v_{1}^{j} & =v_{1}^{j-1}+v_{1}^{j-2} \quad(j \geq 6) ;
\end{aligned}
$$


and

$$
\begin{aligned}
& u_{k}^{j}=u_{k-2}^{j}-u_{k-1}^{j} \quad(k=2,3, \cdots, j) ; \\
& v_{k}^{j}=v_{k-2}^{j}-v_{k-1}^{j} \quad(k=2,3, \cdots, j) .
\end{aligned}
$$

Where $v_{0}^{j}=u_{0}^{j}$ for $j \geq 6$.

By labeling of above, we know $\xi\left(C_{2 j+1}\right)=0(j \geq 6)$.

The labeling of above is illustrated in Fig.4.

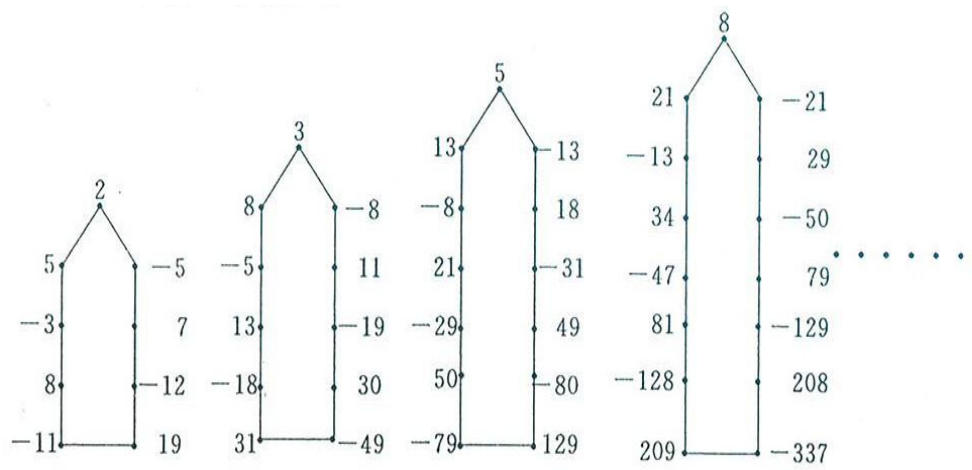

Fig. 4. Labeling of $C_{2 j+1}(j \geq 6)$

Theorem 2.2. For any integer $n \geq 5$, the graph $C_{n}$ is integral sum graph.

Proof. From theorem 2.1, we know $\xi\left(C_{n}\right)=0(n \geq 5)$, therefore for any integer $n \geq 5$, the graph $C_{n}$ is integral sum graph.

\section{References}

1. Harary, F.: Graph Theory. Addison-Wesley, Reading (1969)

2. Bondy, J.A., Murty, U.S.R.: Graph Theory with Applications. Elsevier North-Holland (1976)

3. Harary, F.: Sum graphs over all the integers. Discrete Math. (124), 99-105 (1994)

4. Xu, B.: On integral sum graphs. Discrete Math. (194), 285-294 (1999)

5. Achaya, B.D., Hegde, S.M.: Arithmetic graphs. Journal of Graph Theory 18(3), 275-299 (1990)

6. Yu, H.: The Windmill $W_{n}^{*}$ is the intergral sum Graph and Mod intergral sum Graph. Journal of Heze University 28(3), 18-19 (2006)

7. Gao, X.-L.: Several kinds Interal Sum Tree. Journal of Dezhou University 17(2), 18-22 (2011) 